\title{
The new nutrition label is a combination of the physical activity, traffic light and warning labels: a qualitative study
}

\section{Shirin Seyedhamzeh}

Tehran University of Medical Sciences

\section{Saharnaz Nedjat}

Tehran University of Medical Sciences

\section{Elham Shakibazadeh}

Tehran University of Medical Sciences

\section{Hedayat Hosseini}

Shaheed Beheshti University of Medical Sciences

\section{Azam Doustmohammadian}

Shaheed Beheshti University of Medical Sciences

Ahmad Dorosty ( $\nabla$ dorostim@tums.ac.ir)

Tehran University of Medical Sciences

\section{Research article}

Keywords: nutrition label, food labeling, physical activity

Posted Date: March 31st, 2020

DOl: https://doi.org/10.21203/rs.3.rs-19174/v1

License: (1) This work is licensed under a Creative Commons Attribution 4.0 International License. Read Full License 


\section{Abstract}

Background: Unhealthy diets and inadequate physical activity are important factors in the increasing trend of obesity. In order to mitigate this harmful trend, an effective policy must be developed to target both unhealthy diets and insufficient physical activity. The implementation of physical activity recommendations in nutrition labeling may influence the consumers' food choices. In this qualitative study, we designed a new type of physical activity calorie equivalent (PACE) label by considering the viewpoints of household representatives and experts.

Method: Ten focus group discussions (FGDs) were conducted with 63 mothers from different regions of Tehran based on the participants' socioeconomic status. Furthermore, ten interviews were conducted with food quality control (FQC) experts to examine the new PACE label. The preliminary designs were based on the mothers' viewpoints. We then presented these new labels to the nutritionists and food industry experts in two separate FGDs. After taking their opinions into consideration, we applied certain changes to the labels and emailed them to the mothers. Consequently, the final versions of the new labels were designed. Conventional content analysis was applied with the MAXQDA10 software.

Result: Two main themes were defined: (1) the stakeholders' perspectives on PACE labeling (2) the new PACE label's characteristics including the appearance and the nutritional information on the new label.

Conclusion: The new label is a combination of the physical activity, traffic light and warning labels.

\section{Background}

The increasing prevalence of obesity among the population has become a great challenge. Rapid changes in diets and food behaviors are the causes of this rising trend [1]. Energy imbalances and physical inactivity in children and adolescents are of particular concern [2]. Therefore, in order to mitigate this harmful trend, an effective policy must be developed to target both unhealthy diets and insufficient physical activity [2]. However, these policies may be implemented differently in various countries or in different populations e.g. children [3]. Nutrition labels, taxes, and subsidies on healthy foods are different policy interventions that can target public behavior and the environment. These interventions can take into account different factors such as labeling, price and marketing $[4,5]$.

Nutrition labels are used for both packaged products and restaurant foods. However, there has been much debate on an effective nutrition label that could result in healthier food choices. Various types of numerical and interpretive labels such as nutrition fact, traffic light and star rating labels exist in different countries [6-8]. In fact, there is no consensus among investigators about the impact of nutrition labels on food behavior. Recently, a new type of label that takes physical activity into consideration has been examined in different studies in high income countries [9-13]. This type of label shows the amount of physical activity needed to burn off calories. Thus, this label is a multi-disciplinary policy that considers both calorie content and physical activity. Different studies revealed that using this type of interpretive label helped people to improve their food choices $[1,11,12]$ and caused a limited increase in physical 
activity [14]. However, researches on this type of label have only been conducted in high income countries.

The aim of the present study was to design a new interpretive label based on physical activity. This was achieved by examining the viewpoints of mothers, who represented Iranian households, and industry experts. We also asked the opinions of food quality control (FQC) experts and discussed the feasibility of physical activity calorie equivalent (PACE) labeling.

\section{Methods}

Thematic analysis was applied to determine the main themes. We continued FGDs and interviews until data saturation was achieved. This analysis was part of a larger mixed method study wherein the strengths, weaknesses and strategies of improving the use of nutrition labels were discussed. The stakeholders expressed their opinions, and after careful consideration, a new type of physical activity label was designed. To assess its impact on people's food choices, the new label was then examined in trial groups. The protocol of the present study has been published elsewhere in which the method has been discussed in detail [15]. A flowchart showing the study's processes is presented in figure 1.

\section{Data collection:}

Ten focus group discussions (FGDs) with mothers of schoolchildren between the ages of 6 and 12 years were conducted in ten primary schools of Southern, Northern, Eastern, Western and Central Tehran. The mothers were invited to school by phone. We continued until at least 6 mothers - who met our selection criteria- accepted to participate. Our FGDs were held in two sessions, and were recorded with a voice recorder after obtaining the participants' consents to audio-record them. In the second part of the FGDs, the concept of a PACE label was introduced to the mothers through products such as cake and milk. As these products had different amounts of calories, the suggested physical activity for each label was different. Afterwards, an investigator obtained the necessary information by collecting the participants' opinions about the labels.

After FGDs with the mothers, and while the FGDs findings were being summarized, ten semi-structured interviews about PACE labeling were conducted with FQC experts from different factories which produced dairy products, cakes, biscuits and sweetened beverages. A semi-structured questionnaire was designed by the investigator prior to the interviews. The purposeful method of snowball sampling was applied to select these individuals.

The initial layout of the PACE label was designed by a graphic designer according to the mothers' viewpoints. The mothers' expectations regarding the PACE labels were discussed and the experts' comments were collected. The labels were then changed upon consensus among the researchers and sent to the mothers to outline the final format of the PACE labels. Ultimately, the final versions of the new labels were designed. The complete list of the questions that were asked from the stakeholders during the 
FGDs is presented in table 1. All the sessions were conducted with an investigator who was trained for qualitative studies. Moreover, a note-taker took down all of the comments during the FGDs.

\section{Selection criteria:}

A total of 63 mothers, whose children were 6-12 year old primary school students took part in the study. All participants were literate and willing to participate. Mothers who were nutritionists or food technologists were excluded from the study. However, there were no limitations regarding the age of the participating mothers. The remaining participants, i.e. the FQC and other industry experts were selected by snowball sampling.

\section{Data analysis:}

All of the interviews were transcribed verbatim and typed in Microsoft Word. The MAXQDA 10 software was used to analyze the interviews. The conventional content qualitative analysis approach was applied. The codes were defined and after frequent analysis, two main themes were defined: (1) the stakeholders' perspectives on PACE labeling (2) the new PACE label's characteristics including the appearance and the nutritional information on the new label.

Credibility and conformability of the information were established by extended in-depth engagement with the participants. Prolonged engagement with contributors in the research environment allowed the researcher to realize their trust and a deeper understanding of their situation. All details inclusive of procedures, activities and decisions have been documented for transferability purposes. The reliability and validity of data was confirmed by the second one author.

\section{Ethical considerations:}

This study was approved by Tehran University of Medical Sciences (96-03-161-37037). It was also registered in the Iranian Registry of Clinical Trials (IRCT20181002041201N1). The participants were informed of the research objectives before the interviews. Furthermore, participants were assured that the information would be used only for research purposes and would not be accessible to those who were not in the research team.

\section{Results}

A total of 63 mothers participated in our study. The mothers were $38.54 \pm 5.00$ years old. Their education levels were: school graduates (8.2\%), high school graduates (36.1\%), associates and bachelor's degree graduates (44\%) and graduates of master's and/or higher degrees $(11.7 \%)$.

Interviews with ten FQC experts were carried out. Six of them were educated in food industry, two of them in chemistry and one of them in nutrition. 
FGDs with experts were conducted with 6 PhD graduates of nutrition and 8 PhD graduates of food industry sciences from universities in Tehran.

\section{The stakeholders' perspectives on PACE labeling}

\section{A. Mothers}

Most of the mothers' perspectives on PACE labeling were positive. "If a product has side effects on health, you have the opportunity to know. If you eat a Gaz (an Iranian confection), you should walk for an hour." (33 year old mother)

Some mothers believed that this type of label would change their food choices but not their physical activity habits. However, if weight reduction was an important aspect of their lives, it could motivate them to increase their physical activities. "We have so many problems with our children. When I feel hungry, I eat something and go back to help them with their lessons. I do not have time to burn calories."

Some mothers believed that habits played a more important role in everyday life than time limitations.

"I think that anyone can dedicate an hour a day to exercise and walking. Even employed people can set aside half an hour for walking. It's mostly because of habits, not a busy lifestyle."

"In my opinion, the physical activity required to burn off the calories of let's say a cake, can become a habit. So, after eating a cake or an ice-cream, I will try to burn the calories."

Not all mothers agreed on how these labels would affect their children's choices. Some mothers were certain that they would improve their children's food behavior, while others considered them as ineffective. The reasons that were given for these dissimilar opinions were firstly the children's or their families' habits and secondly the influence of their peers.

\section{B. FQC experts}

Most of the FQC experts provided positive feedbacks on the PACE labels. "I think it would be suitable for people who have weight reduction diets and elders who have a low level of physical activity."

Nevertheless, they believed that pilot studies should certainly be conducted before the implementation of any new type of label. One FQC expert suggested: "You can design a baseline table with information about a physical activity that will burn off 200 calories. At the end of the table you can mention the calorie content."

One FQC expert was clearly opposed to this label. According to this specialist, producers would not cooperate with its application as it would have a strong negative effect on the sales of high calorie products.

\section{The new PACE label's characteristics:}




\section{- The appearance and the nutritional information:}

\section{A. Mothers}

Table 1 shows the characteristics of the new PACE label. The participants unanimously agreed that graphics were more understandable than digits. They also said that walking was the most accessible form of physical activity, as compared to other exercises such as swimming. Colorful labels were highly recommended, especially for children. In addition, most of the mothers believed that presenting both PACE and traffic light labels would be more effective.

"I think physical activity complements the TLL. I mean that if both of them are put together, it will be complete. They both have information about fat, sugar, carbohydrates, calorie content and physical activity which is sufficient."(Figure 2-a)

The participants believed "walking duration" was a more appropriate term than "walking distance".

From the mothers' point of view, every single nutritional fact was important, such as the general and detailed information about the nutrients and the calorie content. However, this may not be possible, because printing all of this data in the form of colorful graphics can affect the labels' ligibility.

Taking the mothers' viewpoints into consideration, with the help of our graphic designer we designed three different physical activity labels by the end of the FGDs ( $b$ in figure 2). We simplified the images by excluding certain variables, such as age and gender to produce an applicable label. In order to convert calories into physical activity, walking at $5.6 \mathrm{~km} / \mathrm{h}$, an average energy consumption of $6.7 \mathrm{kcal} / \mathrm{min}$ and an average weight of $70 \mathrm{~kg}$ were considered for the calculations. For example a package of cake with 228 calories needs 34 minutes brisk walking to burn off. The investigators collectively agreed on the second image. However, the walking image was changed to brisk walking in order to better represent the information (c in figure 2).

\section{B. Experts}

Some of the food industry experts disagreed with physical activity labels on packaged products. They believed that pre-cooked foods and sweets, because of their high calorie content, should be given preference and labeled instead. However, others suggested the PACE information to be included in the nutrition fact table. On the other hand, the nutritionists agreed with the graphic design of the new label and believed it to be easily understood by the public.

All experts believed that using a single color instead of three would simplify matters for the consumer.

"If I were to declare a product as unhealthy based on the TLL colors, I would have used one single color. I would advise against the use of three colors."

The nutritionists believed that consumers ought to be provided with vital, yet concise information. 
"In my opinion, the calorie content must be included. Fat, be it good or bad, is also an important factor as it results in an intake of calories. I believe, considering our society's level of nutrition knowledge, the information that is currently being provided about calories, salt and fat is sufficient."

It was therefore decided to use either one of the green or red colors on the labels. Based on Iran's Food and Drug Administration guidelines [16], if any of the fat, salt or sugar contents of a product are in the red zone (high), the red label will be used. In addition, the clause "high in fat/sugar/salt" along with calorie content, serving size and recommended physical activity will be included on the label. However, if the abovementioned content levels are in the amber (medium) or green (low) zones, only the green label will be used along with calorie content, serving size and recommended physical activity. Regardless of the label's color, the minutes of physical activity required to burn off the calories must be provided.

Finally, the new labels were designed in red and green. The red label will provide extra information about the high amount of fat and/or salt and/or sugar (figure 2-d).

After considering expert opinions, and a consensus among the investigators, the labels were sent to the mothers. They were asked to select their preferred label(s) and/or make changes that would improve their food choices. Thus, the second format of the picture (b) (figure 2) was selected. However, the figurative label was slightly changed as per the mothers opinions. They said that walking is a more popular activity, so people may find the information more applicable to their lives. "The picture suggests running instead of walking, which is a limitation and may not be possible for many women in Iran." (45 year-old mother)

\section{Discussion}

After considering the viewpoints of all stakeholders, the new physical activity label was designed. It is a combination of traffic light, warning [17] and physical activity labels [18]. We used one color (either green or red) to send a message to the consumers; whether to buy the product or not. The label that showed low or medium values of sugar, salt, and fat (defined by the Iranian Food and Drug Administration guidelines) [16] was to be green in color and included information about calorie content, serving size and recommended physical activity. The label with higher than the guideline's values of sugar and/or salt and/or fat was red. In addition, the high value substance would be outlined in a separate clause. Similarly, it provided information about calorie content, serving size and recommended physical activity. As a result, the consumer will see either one of the green or red colors, and will be warned of the harmful content/s. Therefore, this type of label can easily be understood, regardless of the buyer's level of education. The color amber which represents TLL's medium level was omitted to make its printing easier for manufacturers.

The physical activity label was first designed by Swarts et al. [18]. In order to find out its effectiveness, it was examined in other studies for various foods such as fast foods $[9,10,13]$, snacks and beverages [12, 14]. However, these studies' findings were inconsistent [19-21]. Nonetheless, this type of label can improve public awareness about the importance of physical activity in reducing the trend of obesity. It can also lead to a healthier lifestyle among the general population [22, 23]. 
The positive effects of TLLs have been mentioned in different studies [24-26]. However, certain adverse effects such as concerns about eating disorders have been reported [27]. One study suggested that instead of choosing green labels, the participants were more concerned with avoiding red labeled products [28]; this might have happened as a result of using different colors in one product such as biscuits with low sodium (green), moderate fat (amber) and high sugar (red). Nonetheless, we tried to solve this problem by using only one color in the newly designed label.

In Chile and Brazil, a warning label was introduced for certain products that were high in sugar, sodium and saturated fats $[29,30]$. The warning label was designed to prevent the increasing trend of obesity. However, it only provided details about the high amounts of nutrients that had adverse effects on the consumers' health. In our new label, not only high amounts of the abovementioned nutrients are presented, but it also warns the consumers by being red in color. Other extra information such as calorie content, serving size and recommended physical activity provide customers with sufficient details.

\section{Limitations}

During the designing process of a qualitative study, certain inevitable limitations exist. These limitations must be considered when interpreting the findings. Usually, mothers are responsible for the purchase of foodstuff in Iranian households. Therefore, we chose to only interview the schoolchildren's mothers and not their fathers, who may have held entirely different opinions.

We tried to consider every viewpoint presented by the mothers when designing the new label. However, certain ideas such as the use of all-macro fonts and/or the inclusion of micronutrients in the labels were not feasible. On the other hand, with respect to the experts' opinions, we covered more important and comprehensible details. In the new label, we simplified our design by excluding variables such as age and gender. The primary purpose of this study was to design a label which would help people make healthy food choices. This has been achieved by the use of a legible font on a colored label that provides consumers with explicit and concise information.

\section{Conclusions}

Results from the present study suggest that presenting physical activity information alone may not be suitable for everyone. Merging different types of labels into a single label, in order to deliver a consistent message to the consumers in a short time can be a more effective approach toward promoting healthy food purchases. The addition of the newly designed label on product packages could raise awareness among Iranians and help them make healthy food choices.

\section{Abbreviations}

FGD: Focus Group Discussion

PACE: Physical Activity Calorie Equivalent 
FQC: Food Quality Control

\section{Declarations}

\section{Ethics approval and consent to participate}

This study was approved by Tehran University of Medical Sciences with the registry code of 96-03-16137037 and registered at Iranian registry of clinical [trial https://irct.ir/].

At the beginning of the study the consent form was signed by the participants.

\section{Consent for publication}

All authors of this manuscript declared their consent for publication.

\section{Availability of data and material}

The dataset used and analyzed during the current study is available from the corresponding author on a reasonable request.

\section{Competing interests}

None of the authors reported a conflict of interest related to the study. No financial disclosures were reported by the authors of this paper.

\section{Funding}

The study was funded by Tehran University of Medical Sciences.

\section{Authors' contributions}

SS and AD designed research; SS, SN, ESH, HH, AZD and AD conducted research; SS was responsible for data collection. SS, SN and AZD performed data analysis; SS wrote paper; SS, SN, ESH, HH, AZD and AD had primary responsibility for final content. All authors read and approved the final manuscript.

\section{Acknowledgements:}

This research has been supported by Tehran University of Medical Sciences \& health Services grant no. 96-03-161-37037 on 2017-11-26. We hereby thank the school health experts and principals of districts 1 , $2,4,7 \& 18$ and the industrial quality control experts and authorities who helped us in this project.

\section{References}

1. Wolfson, J.A., D.J. Graham, and S.N. Bleich, Attention to physical activity-equivalent calorie information on nutrition facts labels: an eye-tracking investigation. Journal of nutrition education 
and behavior, 2017. 49(1): p. 35-42. e1.

2. Organization, W.H., Global strategy on diet, physical activity and health. 2004.

3. Khan, L.K., et al., Recommended community strategies and measurements to prevent obesity in the United States. Morbidity and Mortality Weekly Report: Recommendations and Reports, 2009. 58(7): p. 1-29.

4. Grier, S. and C.A. Bryant, Social marketing in public health. Annu. Rev. Public Health, 2005. 26: p. 319339.

5. Powell, L.M. and F.J. Chaloupka, Food prices and obesity: evidence and policy implications for taxes and subsidies. The Milbank Quarterly, 2009. 87(1): p. 229-257.

6. Barreiro-Hurlé, J., A. Gracia, and T. De-Magistris, Does nutrition information on food products lead to healthier food choices? Food Policy, 2010. 35(3): p. 221-229.

7. Hamlin, R. and L. McNeill, Does the Australasian "health star rating" front of pack nutritional label system work? Nutrients, 2016. 8(6): p. 327.

8. Seward, M.W., J.P. Block, and A. Chatterjee, A traffic-light label intervention and dietary choices in college cafeterias. American journal of public health, 2016. 106(10): p. 1808-1814.

9. Antonelli, R. and A.J. Viera, Potential effect of physical activity calorie equivalent (PACE) labeling on adult fast food ordering and exercise. PloS one, 2015. 10(7): p. e0134289.

10. Dowray, S., et al., Potential effect of physical activity based menu labels on the calorie content of selected fast food meals. Appetite, 2013. 62: p. 173-181.

11. Hartley, I.E., R.S. Keast, and D.G. Liem, Physical activity-equivalent label reduces consumption of discretionary snack foods. Public health nutrition, 2018. 21(8): p. 1435-1443.

12. Masic, U., P. Christiansen, and E. Boyland, The influence of calorie and physical activity labelling on snack and beverage choices. Appetite, 2017. 112: p. 52-58.

13. Viera, A.J. and R. Antonelli, Potential effect of physical activity calorie equivalent labeling on parent fast food decisions. Pediatrics, 2015. 135(2): p. e376-e382.

14. Deery, C.B., et al., Physical activity calorie expenditure (PACE) labels in worksite cafeterias: effects on physical activity. BMC Public Health, 2019. 19(1): p. 1596.

15. Seyedhamzeh, S., et al., Potential effect of different nutritional labels on food choices among mothers: a study protocol. BMC Public Health, 2020. 20(1): p. 294.

16. Executive guidelines for minimum requirements of labeling food and beverage products. [Persian]. 2015.

17. Corvalán, C., et al., Structural responses to the obesity and non-communicable diseases epidemic: Update on the Chilean law of food labelling and advertising. Obesity reviews, 2019. 20(3): p. 367374.

18. Swartz, J.J., et al., Simplifying healthful choices: a qualitative study of a physical activity based nutrition label format. Nutrition journal, 2013. 12(1): p. 72. 
19. Fenzl, N., K. Bartsch, and J. Koenigstorfer, Labeling exercise fat-burning increases post-exercise food consumption in self-imposed exercisers. Appetite, 2014. 81: p. 1-7.

20. Pang, J. and D. Hammond, Efficacy and consumer preferences for different approaches to calorie labeling on menus. Journal of nutrition education and behavior, 2013. 45(6): p. 669-675.

21. Viera, A.J., et al., Effect of calories-only vs physical activity calorie expenditure labeling on lunch calories purchased in worksite cafeterias. BMC public health, 2019. 19(1): p. 107.

22. Bauman, A. and C.L. Craig, The place of physical activity in the WHO Global Strategy on Diet and Physical Activity. International Journal of Behavioral Nutrition and Physical Activity, 2005. 2(1): p. 10.

23. Lachat, C., et al., Diet and physical activity for the prevention of noncommunicable diseases in lowand middle-income countries: a systematic policy review. PLoS medicine, 2013. 10(6).

24. Machin, L., et al., Traffic Light System Can Increase Healthfulness Perception: Implications for Policy Making. J Nutr Educ Behav, 2018. 50(7): p. 668-674.

25. Cole, M., H. Peek, and D. Cowen, UK consumer perceptions of a novel till-receipt 'traffic-light' nutrition system. Health Promot Int, 2019. 34(4): p. 640-647.

26. Chen, H.J., et al., The application of traffic-light food labelling in a worksite canteen intervention in Taiwan. Public Health, 2017. 150: p. 17-25.

27. Seward, M.W., J.P. Block, and A. Chatterjee, Student experiences with traffic-light labels at college cafeterias: a mixed methods study. Obes Sci Pract, 2018. 4(2): p. 159-177.

28. Scarborough, P., et al., Reds are more important than greens: how UK supermarket shoppers use the different information on a traffic light nutrition label in a choice experiment. Int J Behav Nutr Phys Act, 2015. 12: p. 151.

29. Khandpur, N., et al., Choosing a front-of-package warning label for Brazil: A randomized, controlled comparison of three different label designs. Food Res Int, 2019. 121: p. 854-861.

30. Reyes, M., et al., Development of the Chilean front-of-package food warning label. BMC Public Health, 2019. 19(1): p. 906.

\section{Tables}


Table 1: Questions that were asked from the stakeholders

\begin{tabular}{ll}
\hline Mothers & 1. What is your perspective on PACE labeling? \\
& 2. Do you think it might affect your/child's/children's food \\
& choices? \\
& $\begin{array}{l}\text { 3. Do you think it might affect your/their physical activity? } \\
\text { 4. What should its appearance be like? }\end{array}$ \\
\hline Food Quality Control & 1. What information should it contain? \\
\hline experts & 1. What is your opinion about PACE labeling? \\
& 2. What is your opinion about this label? \\
& 3. What should its appearance be like? \\
& 4. What information should it contain?
\end{tabular}

Table 2: Characteristics of the new label based on the stakeholders' expectations

\begin{tabular}{|c|c|c|}
\hline & Appearance & Information \\
\hline Mothers & $\begin{array}{l}\text { 1. Graphical } \\
\text { features } \\
\text { 2. Colorful } \\
\text { 3. Front-of- } \\
\text { package } \\
\text { 4. Legible }\end{array}$ & $\begin{array}{ll}\text { 1. } & \text { Calorie content } \\
\text { 2. } & \text { Fat } \\
\text { 3. } & \text { Sugar } \\
\text { 4. } & \text { Salt } \\
\text { 5. } & \text { Vitamins and minerals } \\
\text { 6. Age groups } \\
\text { 7. Understandable portion size } \\
\text { 8. } & \text { Minutes of physical activity }\end{array}$ \\
\hline $\begin{array}{l}\text { Nutritionists and food } \\
\text { industry experts }\end{array}$ & $\begin{array}{l}\text { 1. Graphical } \\
\text { features } \\
\text { 2. Single } \\
\text { colored }\end{array}$ & $\begin{array}{l}\text { 1. Brief } \\
\text { 2. Age groups } \\
\text { 3. Highlighting the high amount of } \\
\text { fat, sugar, salt } \\
\end{array}$ \\
\hline
\end{tabular}

\section{Figures}




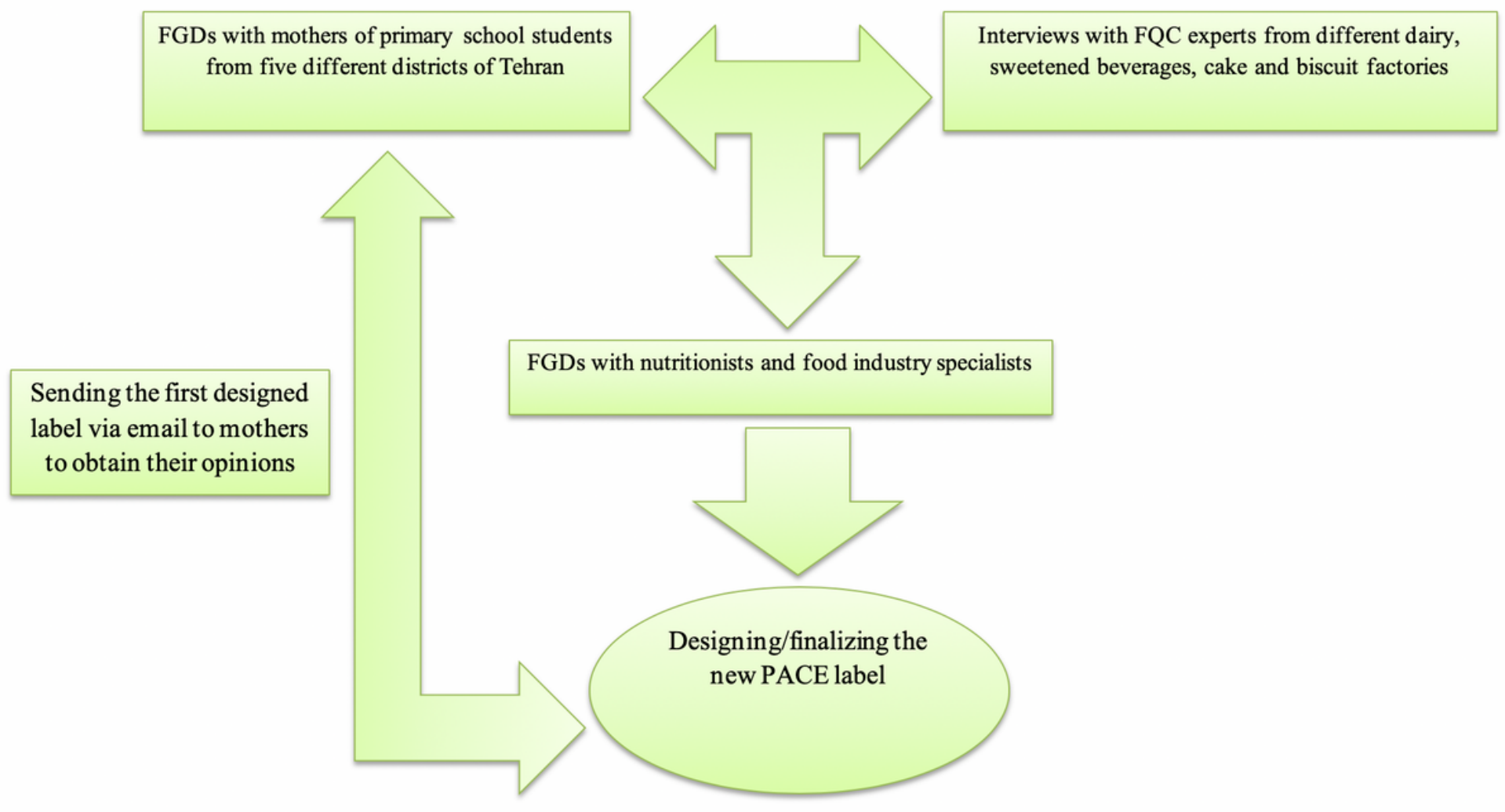

\section{Figure 1}

Study flowchart 
(a)

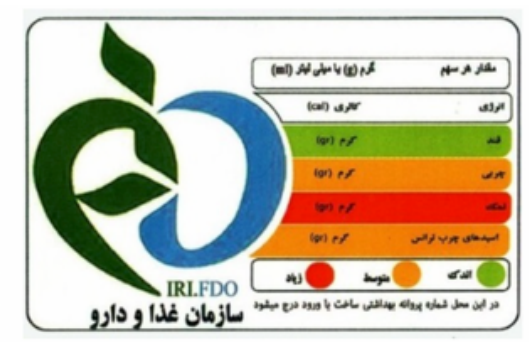

(b)

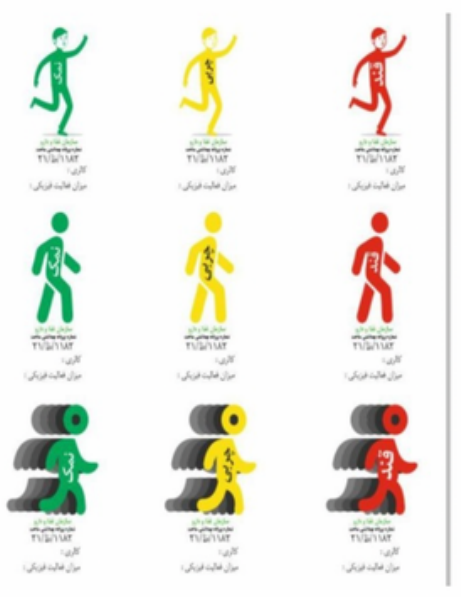

(c)

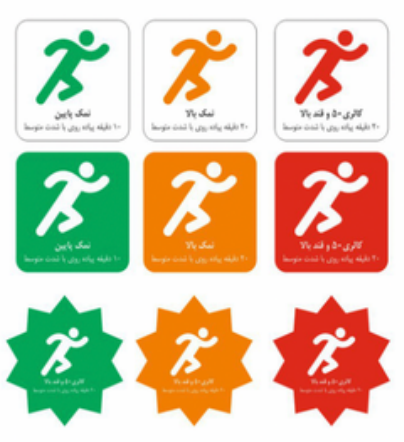

(d)
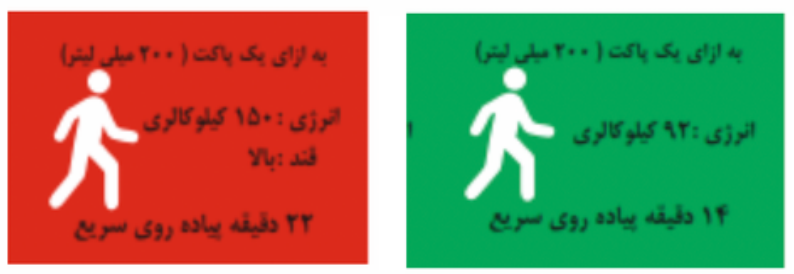

Figure 2

(a) The current traffic light label (b) First edition (c) Second edition (d) The last version of the new label 